\title{
Genome sizes of 227 accessions of Gagea (Liliaceae) discriminate between the species from the Netherlands and reveal new ploidies in Gagea
}

\author{
B J M Zonneveld ${ }^{1 *}$, B te Linde ${ }^{2}$ and L-J van den Berg ${ }^{2}$
}

\begin{abstract}
Nuclear genome size, as measured by flow cytometry with propidium iodide, was used to investigate the relationships within the genus Gagea (Liliaceae), mainly from the Netherlands. The basic chromosome number for Gagea is $x=12$. The inferred ploidy in the Dutch and German accessions varies from diploid to decaploid. Consequently there is a large range of genome sizes (DNA 2C-values) from 14.9 to $75.1 \mathrm{pg}$. Genome sizes are evaluated here in combination with the results of morphological observations. Five species and the hybrid $G$. $\times$ megapolitana are reported. Apart from 14 diploid G. villosa, six plants of G. villosa with an inferred tetraploidy were found. For the 186 Dutch accessions investigated 85 turned out to be the largely sterile G. pratensis (inferred to be pentaploid). Inferred tetraploid and hexaploid G. pratensis were found in 30 and 20 localities, respectively. In one locality an inferred decaploid (10x) plant was found that could represent a doubled pentaploid G. pratensis. An inferred decaploid G. pratensis was never reported before. The genome size of Gagea $\times$ megapolitan a from Germany fitted with its origin as a cross between the two hexaploids G. pratensis and G. lutea. Gagea spathacea from the Netherlands was inferred to be nonaploid as was recorded from plants across Europe. The aim of the study was to use flow cytometry as a tool to elucidate the taxonomic position of the Dutch Gagea.
\end{abstract}

Keywords: Gagea, The Netherlands, DNA 2C-value, Genome size, Ploidy level, New decaploid G. pratensis

\section{Background}

The genus Gagea Salisb. conmprizes about 275 species. In the World Checklist for Gagea (Govaerts 2006) 594 names were listed. It is a genus of small bulbous plants in the family Liliaceae, endemic to Eurasia and North Africa. A single circumpolar species, a former Lloydia is now included in Gagea (Peruzzi 2012). The greatest number of species can be found in Kazakhstan in the Tien Shan and Pamir-Alai. This coincides with the greatest richness of Tulipa (Zonneveld 2010). In Flora Neerlandica (van Oostrom and Reichgelt 1964) four species

\footnotetext{
*Correspondence: ben.zonneveld@naturalis.nl

${ }^{1}$ NBC Naturalis, Herbarium Section, P.O. Box 9517, 2300 RA Leiden, The

Netherlands

Full list of author information is available at the end of the article
}

are recorded for The Netherlands and in Heukels Flora of The Netherlands (van der Meijden 2005) a fifth is added.

To elucidate the relationships between Gagea species, the classical taxonomic traits based on morphological characters, chromosome numbers (Peruzzi 2003, 2012) and sequencing data (Peterson et al. 2008; Zarrei et al. 2009) are here supplemented with data on nuclear DNA content. From only five species genome size was determined earlier (Greilhuber et al. 2000; Vesely et al. 2011; Leitch et al. 2007). Taxonomy of Gagea is rather difficult and the main useful characters so far are: the chromosome numbers, the number and type of bulbils, the number and width of the leaves, the presence of red coloration at the base of the leaf, the hairiness of the flower stalk, the shape of the petals and the number of flowers on a scape. Newer investigations are also based on morpho-anatomical data (Peruzzi 2012).

\section{至 Springer}

(c) 2015 Zonneveld et al. This article is distributed under the terms of the Creative Commons Attribution 4.0 International License (http://creativecommons.org/licenses/by/4.0/), which permits unrestricted use, distribution, and reproduction in any medium, provided you give appropriate credit to the original author(s) and the source, provide a link to the Creative Commons license, and indicate if changes were made. 
186 different accessions from The Netherlands were measured in an attempt to understand the relationships within the Dutch gageas. These values were compared with an additional 41 taxa from Germany. Nuclear DNA content can conveniently be measured by flow cytometry using propidium iodide, a stoichiometric DNA stain that intercalates in the double helix. Where many species in a genus have the same chromosome number, differences in DNA $2 \mathrm{C}$-value have proven to be very effective in delimiting infrageneric divisions in a number of taxa (Ohri 1998). The evolution of genome size (Greilhuber 1979) has received increased attention during recent years (Greilhuber 2005). The smallest angiosperm genome size reported so far is for Genlisia margarethae Hutch. with $2 \mathrm{C}=0.13$ pg (Greilhuber et al. 2006). The record holders for maximum genome size were for eudicots Viscum album L. with $2 \mathrm{C}=205.8 \mathrm{pg}$ and for monocots Paris japonica with $2 \mathrm{C}=304.5$ pg (Pellicer et al. 2010). Flow cytometry was successfully used to measure the $2 \mathrm{C}$-value for the genera Hosta Tratt., Helleborus L., Clivia Lindl., Nerine Herb., Agapanthus L'Hér., Galanthus L., Narcissus L., Gasteria Duval. Tulipa L. etc. by Zonneveld (2001, 2003, 2008, 2009, 2010), Zonneveld and Van Iren (2001), Zonneveld and Duncan (2003, 2006), and Zonneveld et al. (2003, 2012). In this paper it is shown that genome size is helpful to discriminate between the species of Gagea from The Netherlands (Fig. 1).

Nuclear DNA content as measured by using flow cytometry may conveniently be used to produce systematic data. It is applicable even in dormant bulbs or sterile plants for the monitoring of the trade in bulbous species. In the case of Gagea, it is difficult to ascribe a plant to a taxon in the often non-flowering state. Genome size is a good way to determine the species and their ploidy. A different genome size infers usually a different ploidy or a different species. However, the reverse is not true: if plants have the same genome size it does not automatically mean that they are the same species, it might be a coincidence.

Based on van den Berg and te Linde (2003) and new observations, morphological descriptions were given for the species, correlating it with the measured genome weights. New ploidies were inferred for Gagea villosa which, apart from 14 diploids, had six plants with an inferred tetraploid amount of DNA and for G. pratensis that was found to have, apart from the tetraploid, the hexaploid and the very common pentaploid accessions, also a genome size inferring decaploidy.

\section{Methods}

Plant material

The plant material used in this study was collected from locations across The Netherlands and Germany as described in Table 1 . It was mainly obtained from B. te
Linde, Stichting Berglinde, Babberich and a few from L. Duistermaat from NCB Naturalis, Leiden, The Netherlands. Further material came from T. Pfeiffer from the Ernst-Moritz-Arndt-University of Greifswald, Germany. The German plants, supplied with chromosomes counts, were used to infer the ploidy of the Dutch plants. Material of known origin was used. Vouchers will be lodged in the Herbarium of Naturalis Leiden (L). Figures 2, 3, 4, 5, $6,7,8$ show the spread of the taxa in the Netherlands.

\section{Flow cytometric measurement of DNA 2C-value}

For the isolation of nuclei, a few $\mathrm{cm}$ of leaf or a single bulbil was chopped together with a piece of Agave americana L. 'Aureomarginata' or Agave attenuata L. as an internal standard (see below). The chopping was done with a new razor blade in a Petri dish in $0.25 \mathrm{ml}$ nucleiisolation buffer to which $0.25 \mathrm{mg} \mathrm{RNase} / \mathrm{ml}$ was added (Zonneveld and van Iren 2001). After adding $1.75 \mathrm{ml}$ propidium iodide solution (50 mg PI/l in isolation buffer) the suspension with nuclei was filtered through a $20 \mu \mathrm{m}$ nylon filter. The fluorescence of the nuclei at $585 \mathrm{~nm}$ was measured half an hour and $1 \mathrm{~h}$ after addition of propidium iodide excitation, using a BD Accuri C6 flow cytometer equiped with a $488 \mathrm{~nm}$ laser suitable for propidium iodide. Data were analyzed by means of BD Accuri Cflow Plus software provided by the supplier. Plots were first gated to exclude debris on a scatter diagram (Fl2-A vs FL1-A) and counted against FL2-A on a logarithmic scale. The $2 \mathrm{C}$ DNA content of the sample was calculated as the sample peak mean, divided by the Agave peak mean, and multiplied with the amount of DNA of the Agave standard. Two different samples, with each at least 5,000 nuclei, were measured twice for each clone. Most histograms revealed a Coefficient of Variation of less than $5 \%$. The standard deviation was calculated for the DNA content of each species, using all relevant measurements.

\section{Internal standard and absolute DNA content values}

When measuring nuclear DNA content by means of flow cytometry, it is necessary to chop tissue from the plant of interest together with an internal standard. This standard must be as close as possible to the plants of interest and not overlap with the ploidy area of interest. If they are too close together the peak values interfere with each other. Linearity is checked by comparing the different ploidies as found within leaves and roots of many plants. In this way, variation in signal intensities due to staining kinetics, to light absorption and quenching by sample components, as well as to instrument and other variables, is reduced to a minimum. Agave americana was chosen as internal standard for Gagea. For Gagea minima and G. villosa, with 2C-values that more or less coincided with Agave americana, 
Agave attenuata was used. Agave is available yearround, does not mind several weeks without water and, being a large plant, a single specimen can serve a lifetime, thereby further reducing variation in readings. It also has a low background in propidium iodide measurements, and show a single $G_{0}$ peak, almost lacking $G_{2}$ arrest.

Fresh male human leucocytes $[2 \mathrm{C}=7.0 \mathrm{pg}$; $1 \mathrm{pg}=10^{-12} \mathrm{~g}=0.978 \times 10^{9}$ base pairs (Doležel et al. 2003)] were chosen as primary standard (Tiersch et al. 1989). This yields $2 \mathrm{C}=15.9 \mathrm{pg}$ for nuclei of Agave americana L. and $8.0 \mathrm{pg}$ for $A$. attenuata. Based on a published male human genome size of $6.294 \times 10^{9}$ base pairs the nucleus was calculated as containing $6.436 \mathrm{pg}$ (Doležel et al. 2003). However this is based on a human sequence where the size of the very large repeat sequences could not accurately be determined. So in the end the genome size could be closer to $7 \mathrm{pg}$ than now envisioned.

\section{Results}

\section{General}

Morphologically the species of Gagea are rather difficult to differentiate. They are all small bulbous plants with grass like leaves and mostly yellow flowers. Moreover they are visible above the soil surface only about 2 months a year in early spring. The Dutch Gagea can be divided over four out of 12-14 different sections. G. lutea, G. $\times$ pomeranica and G. pratensis belong to section Gagea whereas G. minima, G. villosa and G. spathacea each belong to a separate section. Gageas have been measured from 186 localities in The Netherlands (Tables 1,2) and they are compared with 41 accessions from Germany. They are shown to comprise six taxa with several inferred ploidies.

\section{Gagea minima (L.) Ker Gawl.-section Minimae}

Gagea minima is a small plant with 1 (or 2) narrow 2-3(5) $\mathrm{mm}$ wide leaves and 1-3 flowers per scape. G. minima with $2 \mathrm{C}=14.9 \mathrm{pg}$ from two localities, together with G. villosa, are the only two inferred diploid species found in The Netherlands.

\section{Gagea villosa (M.Bieb.) Sweet-section Didymobolbos}

Gagea villosa is a hairy, largely sterile plant with numerous bulbils. Fourteen accessions of G. villosa from the Dutch provinces of Gelderland, Overijsel and Zuid-Holland are inferred to be diploid with $16.9 \mathrm{pg}$. Six accessions of G. villosa are inferred to be tetraploid with on average $32.3 \mathrm{pg}$. This is based on the basic value of $7-8 \mathrm{pg}$ as in the other species (except G. spathacea) and the published counts of 24 and 48 chromosomes (http://www. tropicos.org/gagea).

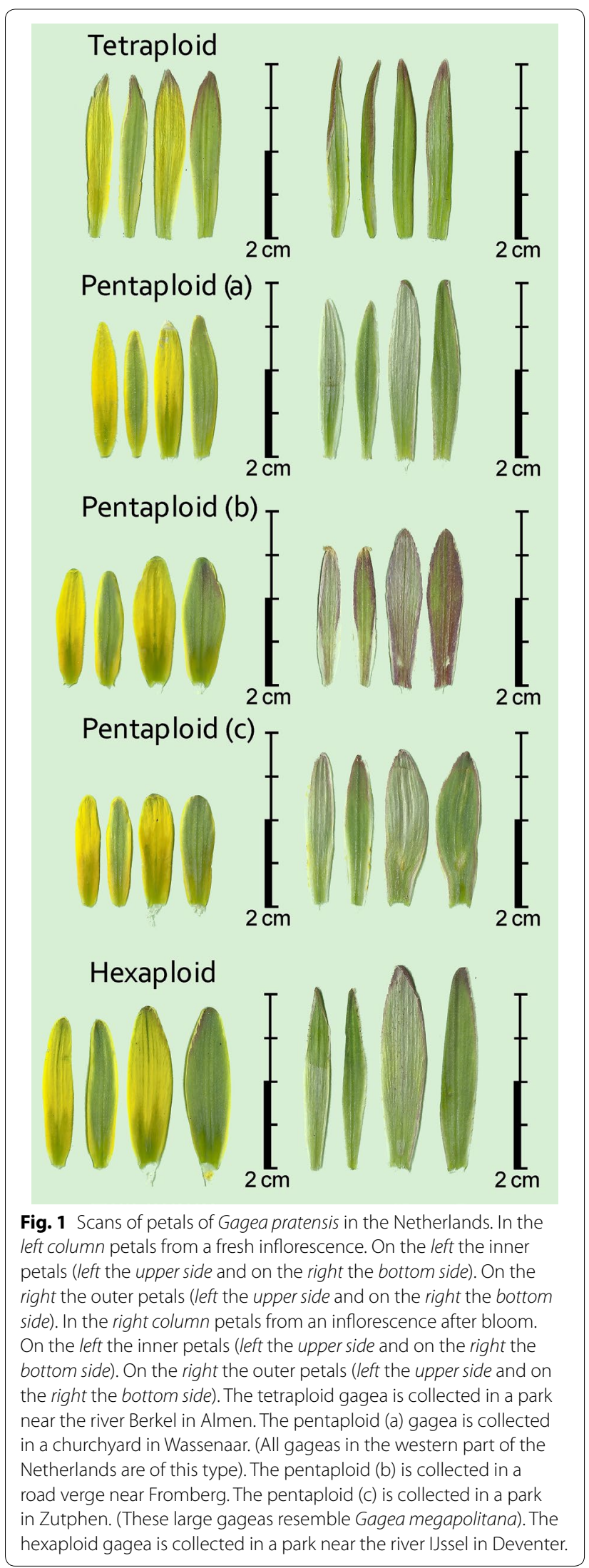


Table 1 Accessions of Gagea from The Netherlands and Germany (in italics), with their nuclear DNA content, inferred ploidy, average, standard deviations and localities

\begin{tabular}{|c|c|c|c|c|c|c|}
\hline Species & $\mathrm{pg} / 2 \mathrm{C}$ & Average & stdev & Locality & coll. $\mathrm{nr}$ & $\begin{array}{l}\text { Locality } \\
\text { (counties NL) }\end{array}$ \\
\hline \multicolumn{7}{|c|}{ Gagea minima diploid } \\
\hline Gagea minima & 14.8 & 14.9 & 0.6 & Leyduin, Bloemendaal & BtL11-0092 & N Holland \\
\hline Gagea minima & 14.9 & & & Leyduin, Bloemendaal & L Duistermaat & N Holland \\
\hline \multicolumn{7}{|c|}{ Gagea villosa diploid } \\
\hline Gagea villosa & 16.5 & 17.0 & 0.4 & Doddendaal & BtL11-053 & Gelderland \\
\hline Gagea villosa & 16.7 & & & Deventer & BtL11-085 & Overijsel \\
\hline Gagea villosa & 16.7 & & & Zutphen, Hanzehof & BtL11-003 & Gelderland \\
\hline Gagea villosa & 16.8 & & & Elten & BtL11-001 & Germany \\
\hline Gagea villosa & 16.8 & & & Eys & BtL11-002 & Limburg \\
\hline Gagea villosa & 16.8 & & & Zevenaar & BtL11-084 & Gelderland \\
\hline Gagea villosa & 16.9 & & & Zeist & BtL11-037 & Utrecht \\
\hline Gagea villosa & 17.0 & & & Lent & BtL11-055 & Gelderland \\
\hline Gagea villosa & 17.0 & & & Valkenburg & BtL11-077 & Limburg \\
\hline Gagea villosa & 17.2 & & & Zevenaar & BtL11-078 & Gelderland \\
\hline Gagea villosa & 17.3 & & & Zalk & BtL11-017 & Overijsel \\
\hline Gagea villosa & 17.4 & & & Mijnsherenland & BtL14-002 & Gelderland \\
\hline Gagea villosa & 17.3 & & & Spijk & BtL14-003 & Gelderland \\
\hline Gagea villosa & 17.2 & & & Amerongen & BtL14-004 & Utrecht \\
\hline \multicolumn{7}{|c|}{ Gagea villosa tetraploid } \\
\hline Gagea villosa & 31.5 & 32.3 & 0.7 & Heelsum & BtL11-052 & Gelderland \\
\hline Gagea villosa & 31.7 & & & Zwolle, Nahuysplein 1 & BtL11-016 & Overijsel \\
\hline Gagea villosa & 31.8 & & & Zwolle, Zandhove & BtL13-079 & Overijsel \\
\hline Gagea villosa & 32.6 & & & Bussloo, begraafplaats t.o Zutphenboer & BtL13-088 & Gelderland \\
\hline Gagea villosa & 32.8 & & & Zwolle, Nahuysplein & BtL13-080 & Overijsel \\
\hline Gagea villosa & 33.2 & & & Azewewijn, kerkhof & BtL13-102 & Gelderland \\
\hline \multicolumn{7}{|c|}{ Gagea pratensis tetraploid } \\
\hline Gagea pratensis & 32.1 & 32.8 & 0.4 & Deventer & BtL11-085 & Overijsel \\
\hline Gagea pratensis & 32.1 & & & Kootwijk & BtL11-073 & Gelderland \\
\hline Gagea pratensis & 32.2 & & & Almen 2 & BtL11-066 & Gelderland \\
\hline Gagea pratensis & 32.2 & & & Legden & BtL13-028 & Germany \\
\hline Gagea pratensis & 32.3 & & & Almen 1 & BtL11-040 & Gelderland \\
\hline Gagea pratensis & 32.3 & & & Rhienderen 1 & BtL11-067 & Gelderland \\
\hline Gagea pratensis & 32.4 & & & Suderas, Wichmond & BtL13-053 & Gelderland \\
\hline Gagea pratensis & 32.4 & & & Bussloo, begraafplaats t.o Zutphenboer & BtL13-087 & Gelderland \\
\hline Gagea pratensis & 32.5 & & & Babberich & BtL11-014 & Gelderland \\
\hline Gagea pratensis & 32.6 & & & Voorst & BtL11-062 & Gelderland \\
\hline Gagea pratensis & 32.7 & & & Gietelo & BtL13-002 & Gelderland \\
\hline Gagea pratensis & 32.7 & & & Ravenswaarden & BtL13-009 & Gelderland \\
\hline Gagea pratensis & 32.7 & & & Keppel, klein & BtL13-050 & Gelderland \\
\hline Gagea pratensis & 32.8 & & & Almen & BtL11-012 & Gelderland \\
\hline Gagea pratensis & 32.9 & & & Trent 2 & Tre11-xx & Germany \\
\hline Gagea pratensis & 32.9 & & & Gingst & Gin11-xx & Germany \\
\hline Gagea pratensis & 32.9 & & & Meppen, stadswal & BtL13-077 & Germany \\
\hline Gagea pratensis & 33.0 & & & Subzow & Sub11-18 & Germany \\
\hline Gagea pratensis & 33.0 & & & Elbe Jasebeck & BtL13-043 & Germany \\
\hline Gagea pratensis & 33.0 & & & Deventer, Brinkgrave & BtL13-084 & Overijsel \\
\hline Gagea pratensis & 33.1 & & & Almen 3 & BtL11-074 & Gelderland \\
\hline Gagea pratensis & 33.2 & & & Zirchow & Zir11-xx & Germany \\
\hline
\end{tabular}


Table 1 continued

\begin{tabular}{|c|c|c|c|c|c|c|}
\hline Species & $\mathrm{pg} / 2 \mathrm{C}$ & Average & stdev & Locality & coll. nr & $\begin{array}{l}\text { Locality } \\
\text { (counties NL) }\end{array}$ \\
\hline Gagea pratensis & 33.2 & & & Deventer, Brinkgrave & BtL13-015 & Overijsel \\
\hline Gagea pratensis & 33.3 & & & Beek & BtL11-045 & Gelderland \\
\hline Gagea pratensis & 33.3 & & & Klarenbeek & BtL11-061 & Gelderland \\
\hline Gagea pratensis & 33.3 & & & Trent & Tre11-xx & Germany \\
\hline Gagea pratensis & 33.3 & & & Kampens dl & BtL13-035 & Overijsel \\
\hline Gagea pratensis & 33.4 & & & Gorssel, oud kerkhof & BtL13-083 & Gelderland \\
\hline Gagea pratensis & 33.7 & & & Bingerden 1a & BtL11-075 & Gelderland \\
\hline Gagea pratensis & 33.7 & & & Gietelo & BtL13-002 & Gelderland \\
\hline \multicolumn{7}{|c|}{ Gagea pratensis pentaploid } \\
\hline Gagea pratensis & 37.9 & 39.9 & 0.5 & Cortenoever 1 & BtL11-087 & Gelderland \\
\hline Gagea pratensis & 37.9 & & & Leyduin & BtL11-041 & N. Holland \\
\hline Gagea pratensis & 38.3 & & & Ressen & BtL11-056 & Gelderland \\
\hline Gagea pratensis & 38.5 & & & Bingerden 2 & BtL11-094 & Gelderland \\
\hline Gagea pratensis & 38.5 & & & Bemmel 1 & BtL11-033 & Gelderland \\
\hline Gagea pratensis & 38.6 & & & Zwolle 2 & BtL11-015 & Overijsel \\
\hline Gagea pratensis & 38.6 & & & Hattem, Heezenberg & BtL13-089 & Gelderland \\
\hline Gagea pratensis & 38.7 & & & Angerlo & BtL11-027 & Gelderland \\
\hline Gagea pratensis & 38.7 & & & Ravenswaarden & BtL11-058 & Gelderland \\
\hline Gagea pratensis & 38.7 & & & Den Haag, Westerduinpark & BtL13-031 & Z. Holland \\
\hline Gagea pratensis & 38.8 & & & Castricum, kerkhof & BtL13-085 & N. Holland \\
\hline Gagea pratensis & 38.9 & & & Didam & BtL13-048 & Gelderland \\
\hline Gagea pratensis & 38.9 & & & Zwolle, Vecht & BtL13-081 & Overijsel \\
\hline Gagea pratensis & 39.0 & & & Loil & BtL11-024 & Gelderland \\
\hline Gagea pratensis & 39.0 & & & Angerlo & BtL11-026 & Gelderland \\
\hline Gagea pratensis & 39.0 & & & Babberich 2 & BtL11-072 & Gelderland \\
\hline Gagea pratensis & 39.0 & & & Den Haag, Marlot & BtL13-032 & Z. Holland \\
\hline Gagea pratensis & 39.0 & & & Lisse, Huis te Spekke & BtL13-024 & Z. Holland \\
\hline Gagea pratensis & 39.1 & & & Haarlem 1a & BtL11-039 & N. Holland \\
\hline Gagea pratensis & 39.1 & & & Leiden, Rhijnhof & BtL13-042 & Z. Holland \\
\hline Gagea pratensis & 39.1 & & & Keppel, groot & BtL13-051 & Gelderland \\
\hline Gagea pratensis & 39.2 & & & Bemmel & BtL11-032 & Gelderland \\
\hline Gagea pratensis & 39.2 & & & Velp 2 & BtL11-029 & Gelderland \\
\hline Gagea pratensis & 39.2 & & & Bemmel 2 & BtL11-032 & Gelderland \\
\hline Gagea pratensis & 39.2 & & & Noordwijk, Gooweg & BZ13-023 & Z. Holland \\
\hline Gagea pratensis & 39.2 & & & Haarlem, Schootersingel & BtL13-036 & N. Holland \\
\hline Gagea pratensis & 39.2 & & & Driehuis begraafplaats & BtL13-037 & N. Holland \\
\hline Gagea pratensis & 39.2 & & & Huize Baak & BtL13-052 & Gelderland \\
\hline Gagea pratensis & 39.2 & & & Deventer, Drouwelerkolk & BtL13-013 & Overijsel \\
\hline Gagea pratensis & 39.2 & & & Hattemerwaard & BtL13-093 & Gelderland \\
\hline Gagea pratensis & 39.2 & & & Velzen, Beeckenstein & BtL13-101 & N. Holland \\
\hline Gagea pratensis & 39.3 & & & Huissen, pastorie & BtL13-040 & Gelderland \\
\hline Gagea pratensis & 39.3 & & & Voorhout & BtL13-022 & Z. Holland \\
\hline Gagea pratensis & 39.3 & & & Eldrik & BtL13-049 & Gelderland \\
\hline Gagea pratensis & 39.3 & & & Kampen, stadswal & BtL13-033 & Overijsel \\
\hline Gagea pratensis & 39.3 & & & Mehr & BtL13-027 & Germany \\
\hline Gagea pratensis & 39.4 & & & Bingerden $1 b$ & BtL11-076 & Gelderland \\
\hline Gagea pratensis & 39.4 & & & Kampen 1a & BtL11-019 & Overijsel \\
\hline Gagea pratensis & 39.4 & & & Brummen 1 & BtL11-068 & Gelderland \\
\hline Gagea pratensis & 39.4 & & & Warmond & BtL13-021 & Z. Holland \\
\hline
\end{tabular}


Table 1 continued

\begin{tabular}{|c|c|c|c|c|c|c|}
\hline Species & $\mathrm{pg} / 2 \mathrm{C}$ & Average & stdev & Locality & coll. nr & $\begin{array}{l}\text { Locality } \\
\text { (counties NL) }\end{array}$ \\
\hline Gagea pratensis & 39.5 & & & Fromberg & BtL11-093 & Limburg \\
\hline Gagea pratensis & 39.5 & & & Oud-Zevenaar & BtL11-023 & Gelderland \\
\hline Gagea pratensis & 39.5 & & & Elten & Elt11-xx & Germany \\
\hline Gagea pratensis & 39.5 & & & Drempt & BtL11-042 & Gelderland \\
\hline Gagea pratensis & 39.5 & & & Zutphen, Hanzehof & BtL13-001 & Gelderland \\
\hline Gagea pratensis & 39.5 & & & Zutphen, kanaal 16 jan 2013 & BtL13-003 & Gelderland \\
\hline Gagea pratensis & 39.5 & & & Epse & BtL13-005 & Gelderland \\
\hline Gagea pratensis & 39.5 & & & Wassenaar & BtL13-006 & Z. Holland \\
\hline Gagea pratensis & 39.5 & & & Sassenheim & BtL13-010 & Z. Holland \\
\hline Gagea pratensis & 39.5 & & & Zutphen, De Hoven & BtL13-011 & Gelderland \\
\hline Gagea pratensis & 39.5 & & & OegstgeesT & BtL13-012 & Z. Holland \\
\hline Gagea pratensis & 39.5 & & & IJmuiden berm & BtL13-038 & N. Holland \\
\hline Gagea pratensis & 39.5 & & & Velzen, Kanaalweg & BtL13-086 & N. Holland \\
\hline Gagea pratensis & 39.5 & & & Achthoven & BtL14-005 & Gelderland \\
\hline Gagea pratensis & 39.6 & & & Haarlem & BtLs.n. & N. Holland \\
\hline Gagea pratensis & 39.6 & & & Hoog-Keppel & BtL11-043 & Gelderland \\
\hline Gagea pratensis & 39.6 & & & Zwolle, Zandhove & BtL13-082 & Overijsel \\
\hline Gagea pratensis & 39.6 & & & Velzen, kerkhof & BtL13-100 & N. Holland \\
\hline Gagea pratensis & 39.6 & & & sdl Zutphen, Hanzehof & BtL14-08 & Gelderland \\
\hline Gagea pratensis & 39.6 & & & sdl Zutphen, Hanzehof & BtL14-008 & Gelderland \\
\hline Gagea pratensis & 39.7 & & & Zutphen & Btl11-022 & Gelderland \\
\hline Gagea pratensis & 39.7 & & & Cortenoever 2 & Btl11-020 & Gelderland \\
\hline Gagea pratensis & 39.8 & & & Beverwijk, Scheybeek & BtL13-039 & N. Holland \\
\hline Gagea pratensis & 39.8 & & & Zwolle, Engelse werk & BtL13-078 & Overijsel \\
\hline Gagea pratensis & 39.9 & & & Hummelo & BtL11-044 & Gelderland \\
\hline Gagea pratensis & 39.9 & & & Leiden, Rhynhof & BZ12-01 & Z. Holland \\
\hline Gagea pratensis & 39.9 & & & Den Haag 2 & BtL11-070 & Z. Holland \\
\hline Gagea pratensis & 40.0 & & & sdl Zutphen, Hanzehof & BtL14-008 & Gelderland \\
\hline Gagea pratensis & 40.0 & & & sdl Zutphen, Hanzehof & BtL14-008 & Gelderland \\
\hline Gagea pratensis & 40.1 & & & Rhienderen 2 & BtL11-067 & Gelderland \\
\hline Gagea pratensis & 40.1 & & & Zutphen, Hanzehof & BtLs.n. & Gelderland \\
\hline Gagea pratensis & 40.2 & & & Cortenoever 3 & BtL11-021 & Gelderland \\
\hline Gagea pratensis & 40.2 & & & Weurt & BtL11-054 & Gelderland \\
\hline Gagea pratensis & 40.2 & & & Heelsum, kerk & BtL14-011 & Gelderland \\
\hline Gagea pratensis & 40.3 & & & Brummen 2 & BtL11-071 & Gelderland \\
\hline Gagea pratensis & 40.3 & & & sdl Zutphen, zwembad & BtL14-009 & Gelderland \\
\hline Gagea pratensis & 40.4 & & & Doesburg & BtL11-028 & Gelderland \\
\hline Gagea pratensis & 40.4 & & & Middachten & BtL11-046 & Gelderland \\
\hline Gagea pratensis & 40.4 & & & parent Zutphen, zwembad & BtL14-010 & Gelderland \\
\hline Gagea pratensis & 40.5 & & & Spankeren & Btl11-060 & Gelderland \\
\hline Gagea pratensis & 40.6 & & & Olburgen & Btl11-047 & Gelderland \\
\hline Gagea pratensis & 40.6 & & & Steenderen & Btl11-048 & Gelderland \\
\hline Gagea pratensis & 40.7 & & & Zutphen, begraaf plaats & BtL13-054 & Gelderland \\
\hline Gagea pratensis & 40.9 & & & Groessen & BtL11-035 & Gelderland \\
\hline Gagea pratensis & 40.9 & & & Oud-Zevenaar & BtL11-057 & Gelderland \\
\hline \multicolumn{7}{|c|}{ Gagea pratensis hexaploid } \\
\hline Gagea pratensis & 43.8 & 45.6 & 1.1 & Zirchow/U 2 & ZiU11-xx & Germany \\
\hline Gagea pratensis & 44.0 & & & Trent 2 & Tre11-xx & Germany \\
\hline Gagea pratensis & 44.3 & & & Vaassen, Canneburg 194/478 & BtL11-095 & Limburg \\
\hline
\end{tabular}


Table 1 continued

\begin{tabular}{|c|c|c|c|c|c|c|}
\hline Species & $\mathrm{pg} / 2 \mathrm{C}$ & Average & stdev & Locality & coll. nr & $\begin{array}{l}\text { Locality } \\
\text { (counties NL) }\end{array}$ \\
\hline Gagea pratensis & 45.0 & & & Bronkhorst, slotheuvel & BtL11-049 & Gelderland \\
\hline Gagea pratensis & 45.1 & & & Deventer, De Worp & BtL13-004 & Overijsel \\
\hline Gagea pratensis & 45.1 & & & Culemborg, Stroomrug & BtL13-008 & Gelderland \\
\hline Gagea pratensis & 45.2 & & & Brummen, Engelenburg & BtL13-017 & Gelderland \\
\hline Gagea pratensis & 45.3 & & & Oosterbeek, gazon & BtL11-051 & Gelderland \\
\hline Gagea pratensis & 45.4 & & & Meppen begraafplaats & BtL13-072 & Germany \\
\hline Gagea pratensis & 45.4 & & & Deventer, Blauwijk & BtL13-014 & Overijsel \\
\hline Gagea pratensis & 45.4 & & & Marle, uiterwaardgrasland & BtL13-090 & Overijsel \\
\hline Gagea pratensis & 45.7 & & & Brummen, Ganzenei & BtL13-091 & Gelderland \\
\hline Gagea pratensis & 45.8 & & & Meppen stadswal & BtL13-071 & Germany \\
\hline Gagea pratensis & 46.0 & & & Elst, Johan de Wittstraat & BtL11-030 & Gelderland \\
\hline Gagea pratensis & 46.0 & & & Altenkirchen & Alt11-19 & Germany \\
\hline Gagea pratensis & 46.1 & & & Olst, gementehuis & BtL13-016 & Overijsel \\
\hline Gagea pratensis & 46.6 & & & Ravenswaarden & BtL13-099 & Gelderland \\
\hline Gagea pratensis & 47.2 & & & Empe & BtL11-063 & Gelderland \\
\hline Gagea pratensis & 47.4 & & & Brummen 3 & BtL11-064 & Gelderland \\
\hline Gagea pratensis & 47.9 & & & Brummen 4 & BtL11-069 & Gelderland \\
\hline \multicolumn{7}{|l|}{ Gagea pratensis decaploid } \\
\hline G.pratensis & 75.0 & 75.8 & 1.1 & Kampen $1 \mathrm{~b}$ & BtL11-018 & Overijsel \\
\hline G.pratensis & 76.6 & & & Kampen 1c & BtL11-060 & Overijsel \\
\hline \multicolumn{7}{|c|}{ Gagea $\times$ megapolitana hexaploid } \\
\hline Gagea $\times$ megapolitana & 46.7 & 46.8 & 0.1 & Gingst 2 & Gin 11-xx & Germany \\
\hline Gagea $\times$ megapolitana & 46.8 & & & Meppen & BtL13-071 & Germany \\
\hline \multicolumn{7}{|l|}{ Gagea lutea hexaploid } \\
\hline Gagealutea & 41.7 & 42.7 & 0.6 & Trent 1 & DEs.n. & Germany \\
\hline Gagealutea & 41.9 & & & Haarlem, Spaem en Hout & BtL11-039 & N. Holland \\
\hline Gagealutea & 42.1 & & & Epe, Dinkel & BtL13-030 & Germany \\
\hline Gagealutea & 42.1 & & & Vreden, Berkel & BtL13-029 & Germany \\
\hline Gagealutea & 42.1 & & & Miste & BtL13-103 & Gelderland \\
\hline Gagealutea & 42.3 & & & Roden & BtL11-081 & Drenthe \\
\hline Gagealutea & 42.3 & & & Kelmis, Hohntal & BtL11-079 & Belgie \\
\hline Gagealutea & 42.4 & & & Zuidlaren & BtL11-089 & Drenthe \\
\hline Gagealutea & 42.4 & & & Griebenow & DE-s.n. & Germany \\
\hline Gagealutea & 42.6 & & & Meppen, stadswal & BtL13-070 & Germany \\
\hline Gagealutea & 42.6 & & & Bron Berkle & Bbe11-xx & Germany \\
\hline Gagealutea & 42.6 & & & Bredevoort & BtL11-011 & Gelderland \\
\hline Gagealutea & 42.8 & & & Haarlem 1c & BtL11-038 & N. Holland \\
\hline Gagealutea & 42.8 & & & D Meppen & BtL13-026 & Germany \\
\hline Gagealutea & 42.8 & & & Millingerwaard & BtL14-001 & Z. Holland \\
\hline Gagealutea & 42.9 & & & Gesher & Ges11-xx & Germany \\
\hline Gagealutea & 42.9 & & & Haarlem, Spaem en Hout & BtL11-38 & N. Holland \\
\hline Gagealutea & 42.9 & & & Elbe, Jasebeck & BtL13-044 & Germany \\
\hline Gagealutea & 43.2 & & & Aerdenhout & BtL13-066 & N. Holland \\
\hline Gagealutea & 43.4 & & & Winterswijk, Vreehorst weg & BtL13-055 & Gelderland \\
\hline Gagealutea & 43.8 & & & Bronkhorst & BtL11-005 & Gelderland \\
\hline Gagealutea & 43.9 & & & Schoorl & BtL13-094 & N. Holland \\
\hline Gagea lutea var. glauca & 41.9 & 42.3 & 0.3 & Anloo, grasland & BtL13-064 & Drenthe \\
\hline Gagea lutea var. glauca & 41.9 & & & Veenhof, Berm & BtL13-057 & Drenthe \\
\hline Gagea lutea var. glauca & 42.0 & & & Groningen, Noorderplantsoen & BtL13-045 & Groningen \\
\hline
\end{tabular}


Table 1 continued

\begin{tabular}{|c|c|c|c|c|c|c|}
\hline Species & $\mathrm{pg} / 2 \mathrm{C}$ & Average & stdev & Locality & coll. nr & $\begin{array}{l}\text { Locality } \\
\text { (counties NL) }\end{array}$ \\
\hline Gagea lutea var. glauca & 42.1 & & & Zeegse & BtL11-090 & Drenthe \\
\hline Gagea lutea var. glauca & 42.1 & & & Bellingwolde, berm & BtL13-067 & Groningen \\
\hline Gagea lutea var. glauca & 42.2 & & & Midwolda, Ennemaborg & BtL13-047 & Groningen \\
\hline Gagea lutea var. glauca & 42.2 & & & Eext Berm & BtL13-058 & Drenthe \\
\hline Gagea lutea var. glauca & 42.2 & & & Westeresch & BtL11-080 & Drenthe \\
\hline Gagea lutea var. glauca & 42.3 & & & Sterrenbospark & BtL11-088 & Groningen \\
\hline Gagea lutea var. glauca & 42.3 & & & Gieten & BtL11-009 & Drenthe \\
\hline Gagea lutea var. glauca & 42.3 & & & Doetinchem, Zumpe 16 jan 2013 & BtL13-007 & Gelderland \\
\hline Gagea lutea var. glauca & 42.4 & & & Midlaren & BtL13-059 & Drenthe \\
\hline Gagea lutea var. glauca & 42.8 & & & Wedde, Huis Te Wedde, onder linde & BtL13-069 & Groningen \\
\hline Gagea lutea var. glauca & 42.8 & & & Appingedam, Ekenstein & BtL13-046 & Groningen \\
\hline Gagea lutea var. glauca & 43.0 & & & Eext, Brink & BtL13-056 & Drenthe \\
\hline Gagea lutea var. glauca & 42.9 & & & Naumburg & BtL14-012 & Germany \\
\hline \multicolumn{7}{|c|}{ Gagea $\times$ pomeranica pentaploid } \\
\hline Gagea $\times$ pomeranica & 34.2 & 34.9 & 0.6 & Vitense & Vit11-11 & Germany \\
\hline Gagea $\times$ pomeranica & 34.3 & & & Zirchow/U & ZIU11-XX & Germany \\
\hline Gagea $\times$ pomeranica & 34.3 & & & Zirchow/U 2 & ZIU11-XX & Germany \\
\hline Gagea $\times$ pomeranica & 34.4 & & & W. Baggendorf & WBa11-15 & Germany \\
\hline Gagea $\times$ pomeranica & 34.8 & & & Zirchow/U 2 & ZiU11-Xx & Germany \\
\hline Gagea $\times$ pomeranica & 35.1 & & & Semlow 2 & Sem11-xx & Germany \\
\hline Gagea $\times$ pomeranica & 35.2 & & & Semlow & Sem11-xx & Germany \\
\hline Gagea $\times$ pomeranica & 35.2 & & & Poseritz & Pos11-12 & Germany \\
\hline Gagea $\times$ pomeranica & 35.3 & & & Semlow 2 & Sem11-xx & Germany \\
\hline Gagea $\times$ pomeranica & 35.5 & & & Zirkow/R & ZiR11-12 & Germany \\
\hline Gagea $\times$ pomeranica & 36.0 & & & Semlow & Sem11-xx & Germany \\
\hline \multicolumn{7}{|l|}{ Gagea spathacea nonaploid } \\
\hline Gagea spathacea & 45.4 & 46.7 & 0.8 & Ootmarsum, de Voort & BtL13-063 & Z. Holland \\
\hline Gagea spathacea & 45.4 & & & Zeegse & BtL11-091 & Drenthe \\
\hline Gagea spathacea & 45.5 & & & Losser & BtL11-082 & Overijsel \\
\hline Gagea spathacea & 45.9 & & & Samerot, eiken-haagbeukenbos & BtL13-074 & Germany \\
\hline Gagea spathacea & 46.1 & & & Vasse, beekoever & BtL13-076 & Overijsel \\
\hline Gagea spathacea & 46.1 & & & Brummen, Ganzenei, stroomrug & BtL13-092 & Gelderland \\
\hline Gagea spathacea & 46.3 & & & Amen & BtL14-006 & Drenthe \\
\hline Gagea spathacea & 46.4 & & & Bentheim, langs pad & BtL13-073 & Germany \\
\hline Gagea spathacea & 47.0 & & & Roden, Havezate & BtL11-081 & Drenthe \\
\hline Gagea spathacea & 47.0 & & & Peizermade, bosrand & BtL13-096 & Drenthe \\
\hline Gagea spathacea & 47.2 & & & Roden, Havezate & BtL13-097 & Drenthe \\
\hline Gagea spathacea & 47.3 & & & Wüllen, eiken-haagbeukenbos & BtL13-068 & Germany \\
\hline Gagea spathacea & 47.5 & & & Nietap & BtL13-098 & Drenthe \\
\hline Gagea spathacea & 47.6 & & & Bentheim, gazon & BtL13-041 & Germany \\
\hline Gagea spathacea & 47.7 & & & Ootmarsum, weilandrand & BtL13-075 & Overijsel \\
\hline Gagea spathacea & 47.9 & & & Varik, eikenbos & BtL13-065 & Drenthe \\
\hline Gagea spathacea & 48.1 & & & Peize & BtL13-095 & Drenthe \\
\hline
\end{tabular}

All were measured against Agave americana, but for G. minima and G. villosa A. attenuta was used.

BtL B. te Linde, stdev standard deviation, coll.nr collection number. 


\section{Gagea pratensis (Pers.)Dumort.-section Gagea}

Gagea pratensis is a glabrous plant with up to four flowers per scape. Characteristic are the two nude egglike, horizontal bulbils. Gagea pratensis can be found in The Netherlands with four different inferred ploidies. They can be recognized in that the tetraploid has the leaf sheath circling the stem halfway, the pentaploid three-quarter and the hexaploid and the decaploid completely. They are shown in Figs. 9, 10, 11, 12 and 13. The tetraploids (30 accessions) have a DNA 2C-value (nuclear DNA content) of on average $32.8 \mathrm{pg}$, the pentaploids (85 accessions) have on average $39.9 \mathrm{pg}$ and the hexaploids (20 accessions) have on average $45.6 \mathrm{pg}$. The pentaploids could be hybrids between the tetraploid and the hexaploid cytotypes. Even a decaploid with $75.8 \mathrm{pg}$ has been found. The pentaploid form of G. pratensis is by far the most common Gagea in The Netherlands with 39.5 pg from 85 out of 186 localities. The same ploidy is counted in all 7 populations of $G$. pratensis from Mecklenburg (Germany) (Henker 2005). Therefore it seems most likely that the decaploid plant is derived from the frequently found pentaploid $G$. pratensis that has in this case doubled its genome. As often in polyploids, DNA might have been lost and a similar loss is found in the hexaploid G. pratensis but not in the lower ploidies. The inferred decaploid plants have not been reported before for G. pratensis. Being pentaploid in most cases, it comes as no surprise that G. pratensis is considered to be sterile (van der Meijden 2005). Taxa with anorthoploid chromosome sets

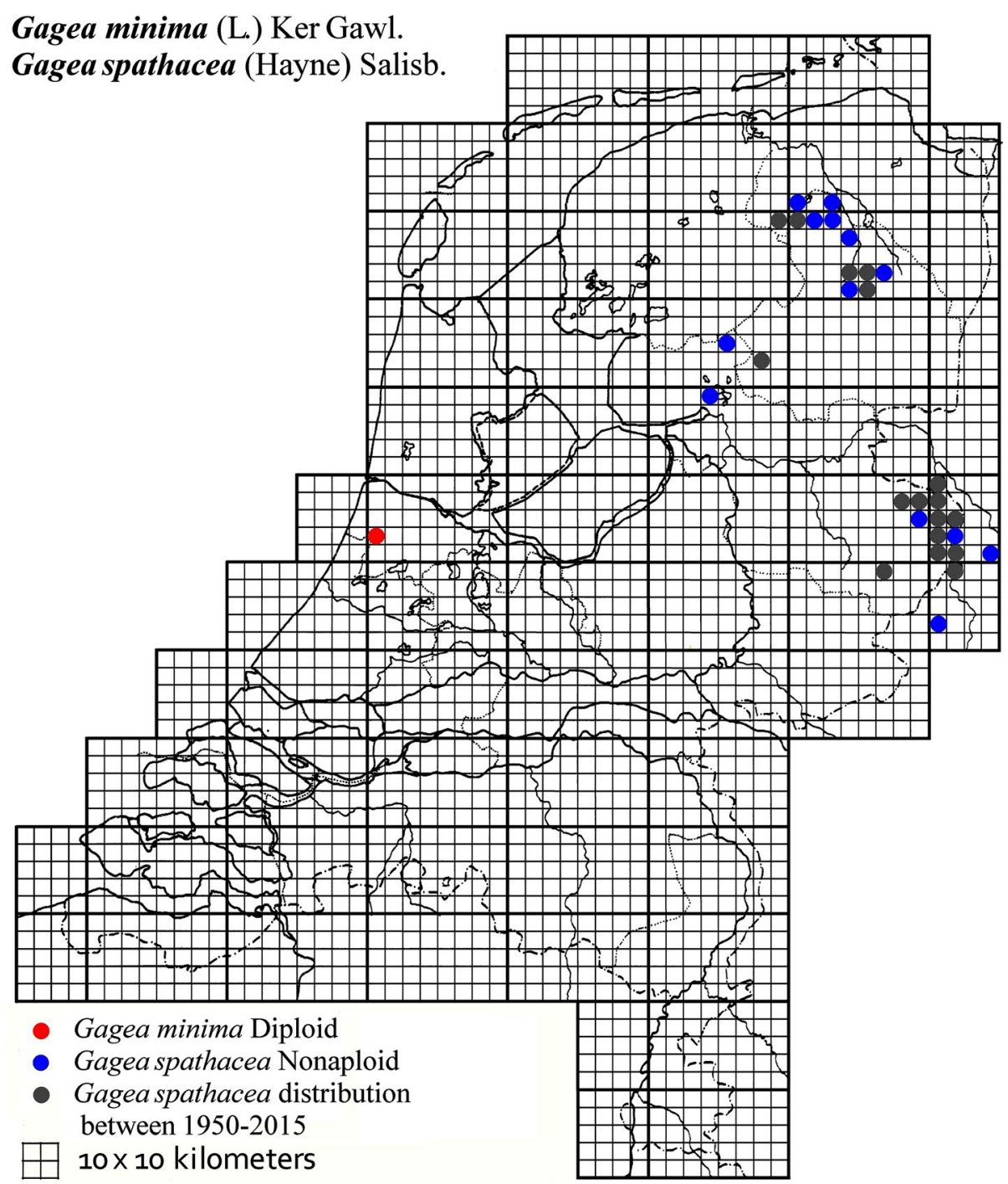

Fig. 2 The distribution of Gagea minima and Gagea spathacea in the Netherlands. 


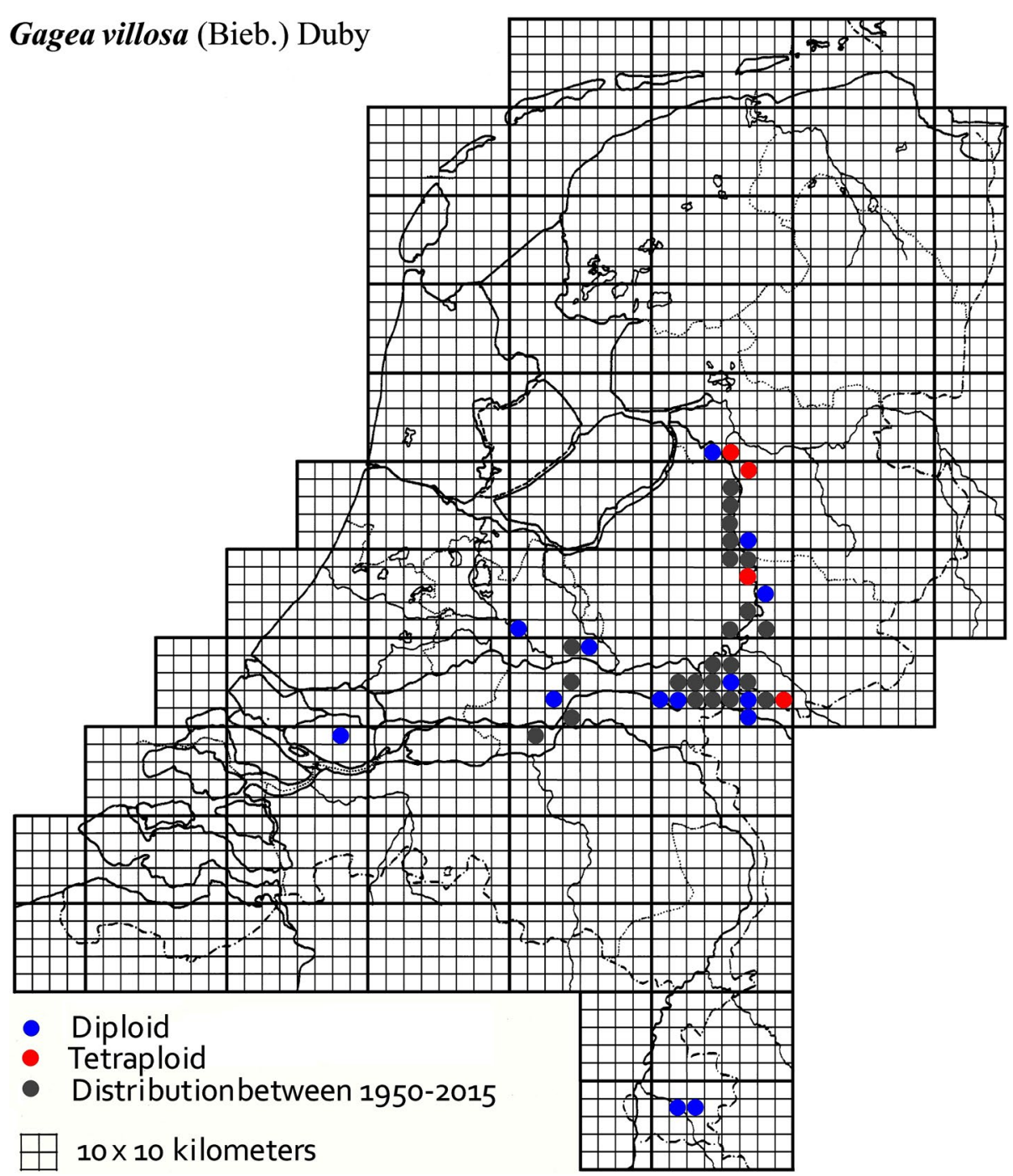

Fig. 3 The distribution of Gagea villosa in the Netherlands.

often show a highly irregular meiosis. An exception are large plants from Zutphen, NL that are fully fertile and differ morphologically with a large basal leaf and 4-8 flowers to a stem. They have a genome size similar to pentaploid G. pratensis, but look more like G. × megapolitana Henker (Henker 2005). Out of 50 germinated seeds, five seedlings measured from the Zutphen locality had the same genome size as their parents. This is peculiar for a pentaploid. Earlier analysis of seedlings of the triploid Hosta 'Sum and Substance' show different, but lower genome sizes in the seedlings (Zonneveld and Pollock 2012). Pfeiffer et al. (2013) report also that some pentaploid populations of G. pratensis are partially fertile. None of the calculated genome sizes of the possible hybrids between G. lutea and $G$. pratensis would fit the plants from Zutphen. Hence more research is required to explain these results.

\section{Gagea lutea (L.) Ker Gawl.-section Gagea}

Gagea lutea is a glabrous plant with leaves of more than $1 \mathrm{~cm}$ wide and up to seven flowers per stem. The bulbs have a diameter of $0.75-1.5 \mathrm{~cm}$ and form numerous bulbils.

Apart from hexaploid G. pratensis also G. lutea is inferred to be hexaploid with $2 \mathrm{C}=42.7 \mathrm{pg}$, collected in 22 localities. Gagea lutea var. glauca (a synonym of $G$. 


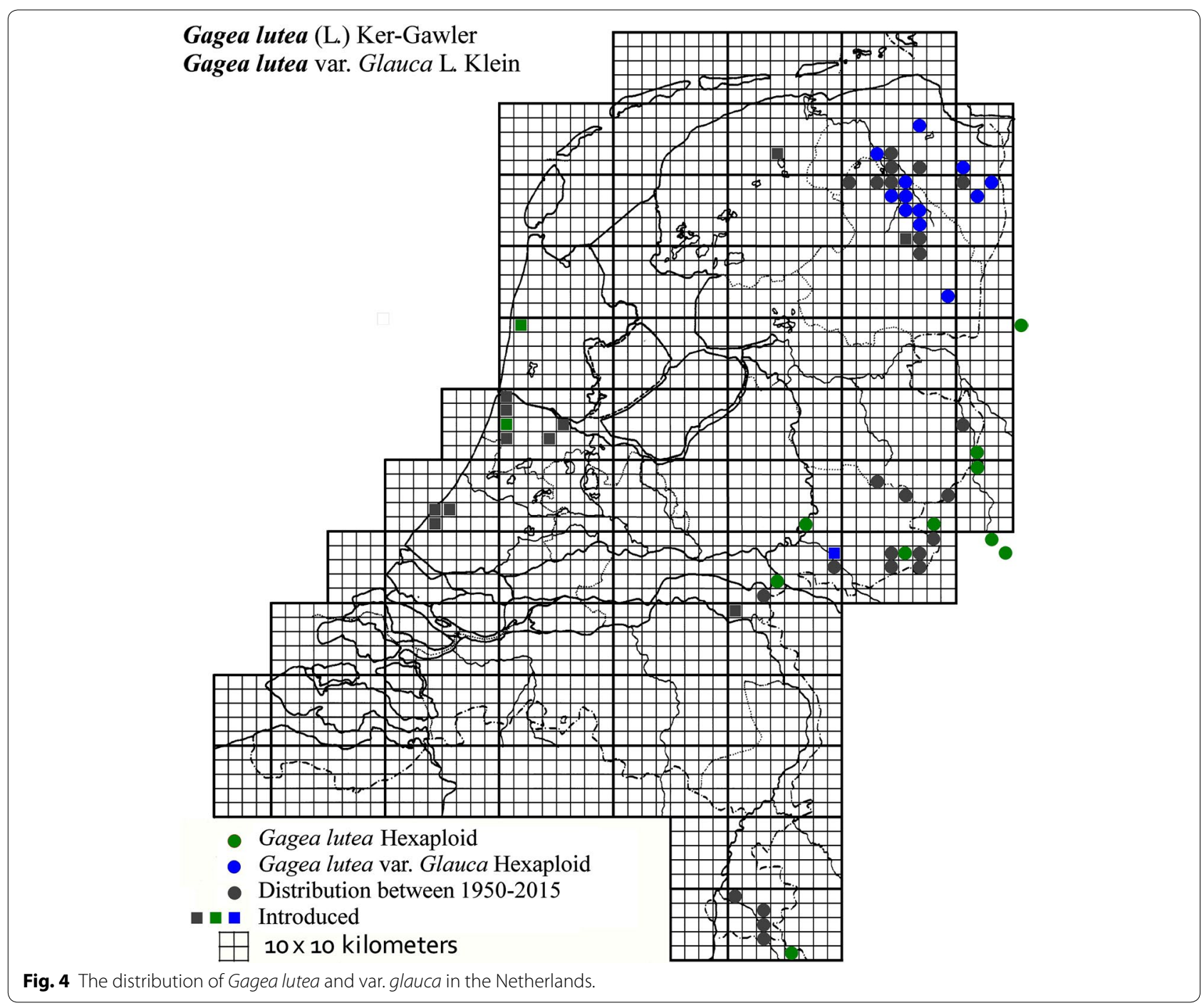

lutea (L.)Ker Gawl.) differs in its glaucous leaves, slightly larger petals, lower fertility and the anthropogenic habitats it grows in. The flowering time of the glaucous forms starts about 2 weeks later when transplanted in the garden. Gagea var. glauca is restricted to the northern part of the Netherlands and is found in localities separate from the green-leaved form. However, with $42.3 \mathrm{pg}$ for 16 different accessions there is no significant difference in genome size.

\section{Gagea spathacea(Hayne) Salisb.-section Spathaceae}

Gagea spathacea is a glabrous plant with 1-3 flowers per stem and is usually found in fairly moist places. Gagea spathacea is only present as a nonaploid plant across (Eastern) Europe (Westergaard 1936; Henker 2005; Pfeiffer et al.
2012). It is observed in about 70 localities in The Netherlands, of which material was collected at 12 localities with an average of $2 \mathrm{C}=46.5 \mathrm{pg}$. This implies a low basic $(\mathrm{Cx})$ value of 5.2, instead of 7.4-8.4 for the other three species. The virtually sterile G. spathacea (Pfeiffer et al. 2012) seems to be a nearly monoclonal plant able to occupy a significant range by dispersal of bulbils (Pfeiffer et al. 2011; 2013).

\section{Hybrid species}

Hybridization and polyploidy are amongst the most important evolutionary mechanism in plants. The parents can be deduced by comparing the genome size of possible parents and their offspring. If parents have say 20 and $30 \mathrm{pg}$ then their offspring will mostly have $25 \mathrm{pg}$. In more complicated allopolyploids the contribution of 


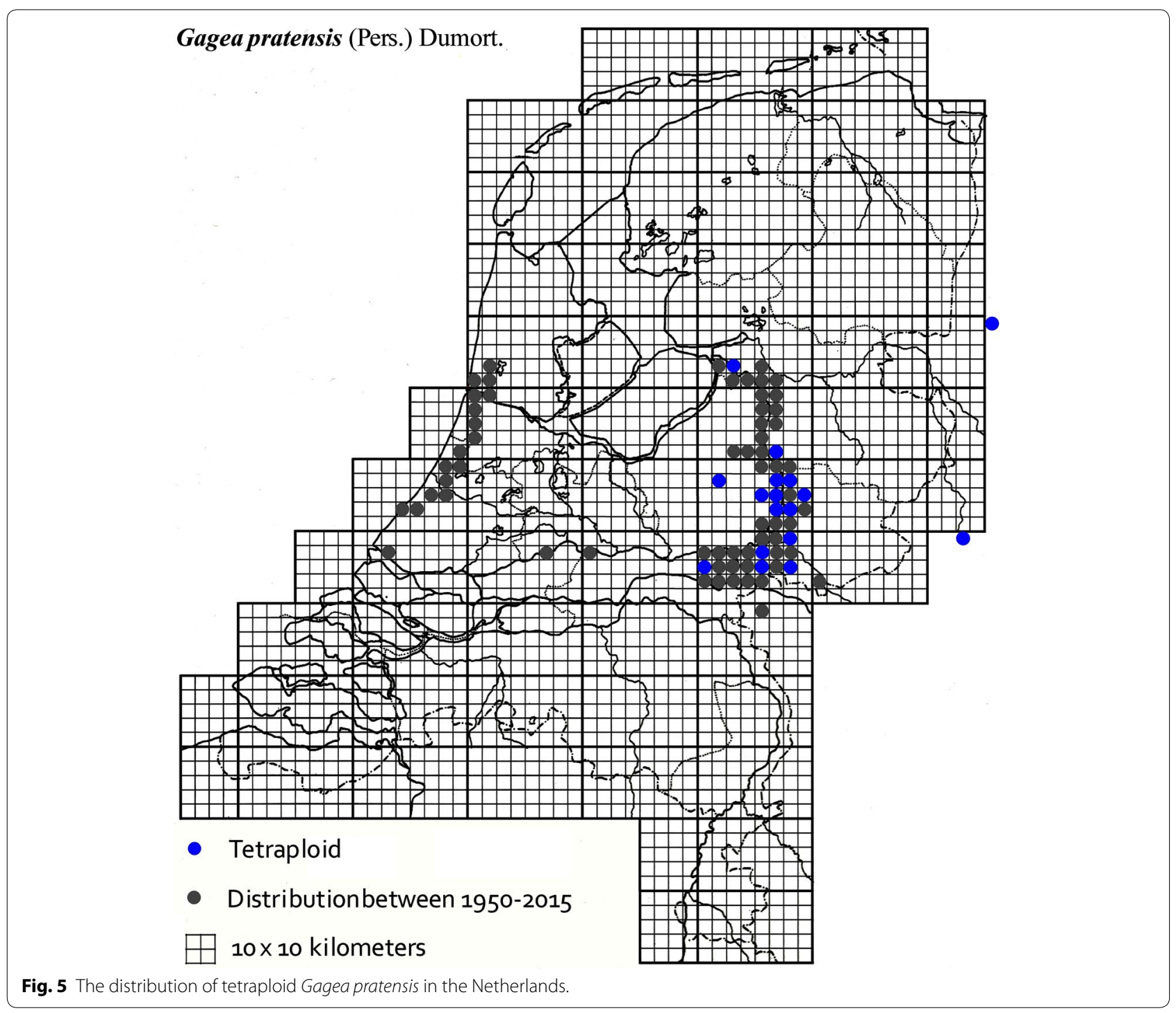

each parent can often be calculated. In Gagea inferred polyploids run from triploid to decaploid (Peruzzi 2003) whereby several species show different ploidies (Henker 2005). Three different hybrids have been described that have the same parents, G. lutea and G. pratensis but combining different ploidies. These three hybrids are here discussed under the names as found in the literature. They are not found in The Netherlands, but two of the hybrids were obtained from Germany (Table 1). The hybrids mostly occur in anthropogenically disturbed sites like churchyards, parks and marginally used meadows. Their parent species are found in forests (G. lutea) and forest edges (G. pratensis). Based on maternal inheritance of the plastids G. pratensis provide the female gametes for $G$. $\times$ pomeranica and G. megapolitana (Peterson et al. 2009).

\section{Gagea $\times$ pomeranica R.Ruthe}

The pentaploid G. $\times$ pomeranica (R.Ruthe) Henker with two genomes of the tetraploid G. pratensis and three genomes of the hexaploid G. lutea (Peterson et al. 2009). However, in the case of G. $\times$ pomeranica, 11 accessions were obtained from Germany that had on average a nuclear DNA content of $34.9 \mathrm{pg}$. This differs considerably (2.2 pg) from the calculated genome size of $37.1 \mathrm{pg}$, based on the basic values for G. lutea and G. pratensis. One explanation could be that this hybrid is an old one and has lost DNA. Another possibility is that other species are involved. Pfeiffer et al. (2013) have shown that backcrosses of the hybrid, mostly with the fully fertile hexaploid G. lutea as pollen parent are possible. However, backcrosses of $G$. × pomeranica (34.9 pg) with $G$. 


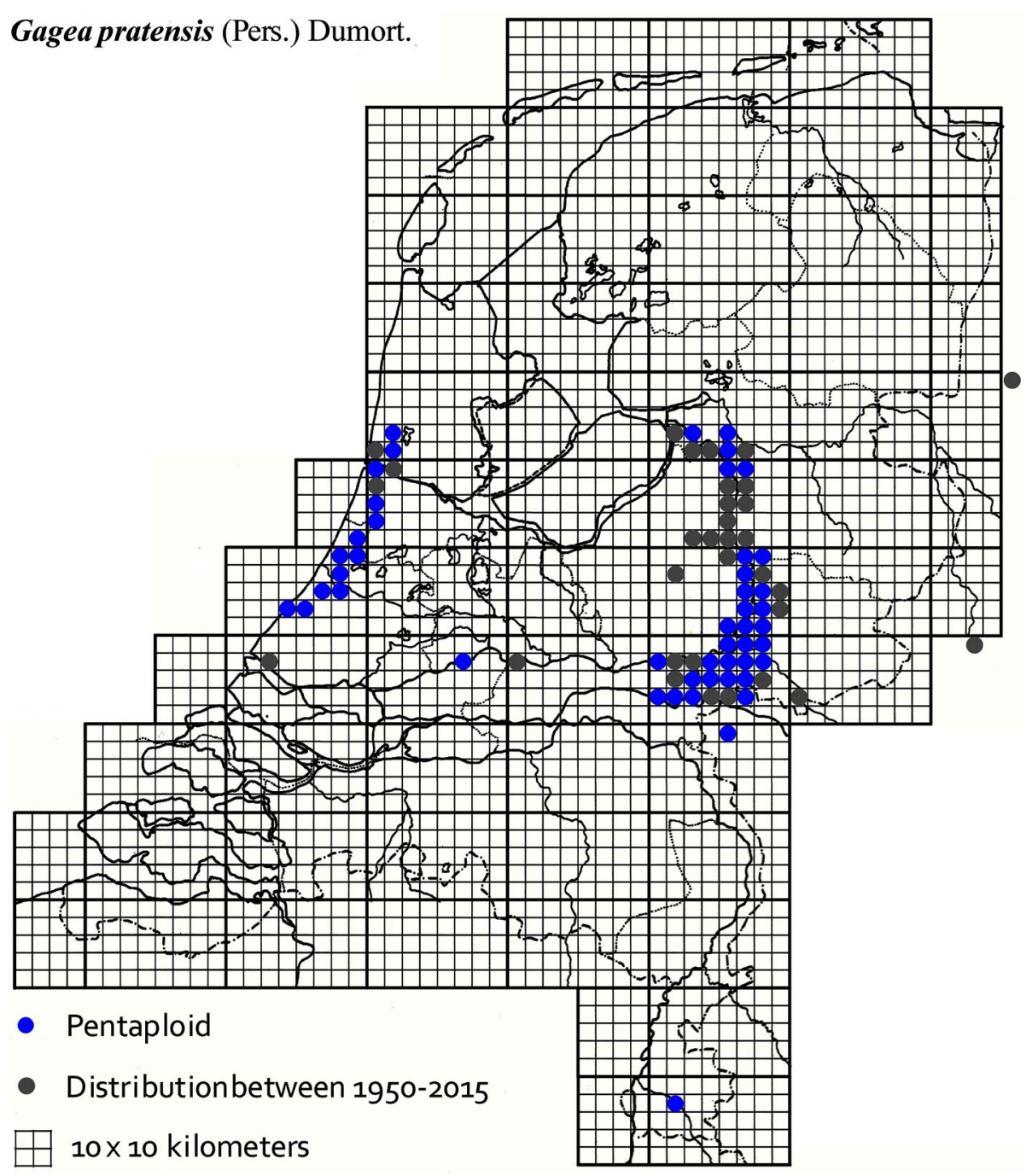

Fig. 6 The distribution of pentaploid Gagea pratensis in the Netherlands.

lutea (42.6 pg) might give higher $2 \mathrm{C}$-values not lower, but these were not observed.

\section{Gagea x marchica Henker. Kiesew., U.Raabe, Rätzel}

Recently another sterile pentaploid hybrid was described as G. marchica Henker et al. (2012) It is described as falling morphologically between the pentaploid $G .(\times)$ pomeranica and the hexaploid $G .(\times)$ megapolitana with 57,59 but probably 60 chromosomes. If it is supposed to be the reversed hybrid (compared to the parents of $G \times$ pomeranica) between hexaploid G. pratensis and tetraploid G. lutea the problem arises that a tetraploid $G$. lutea has not been reported so far (Pfeiffer et al. 2013).

\section{Gagea $\times$ megapolitana Henker}

A third hybrid with the same parents is the hexaploid G. $\times$ megapolitana Henker with three genomes of the hexaploid G. pratensis and three genomes of the hexaploid G. lutea (Peterson et al. 2009). It was obtained from two localities in Germany with on average $2 \mathrm{C}=46.8 \mathrm{pg}$. In the world checklist for monocots (Govaerts 2006) G. megapolitana is accepted as a species. However Peterson et al. (2009) have clearly shown that it is a hybrid between the hexaploids G. pratensis and G. lutea. The genome size provides a firm argument for this hybridity and confirm the suggestion of Peterson et al. (2009) for the parents and the ploidy of G. × megapolitana. 


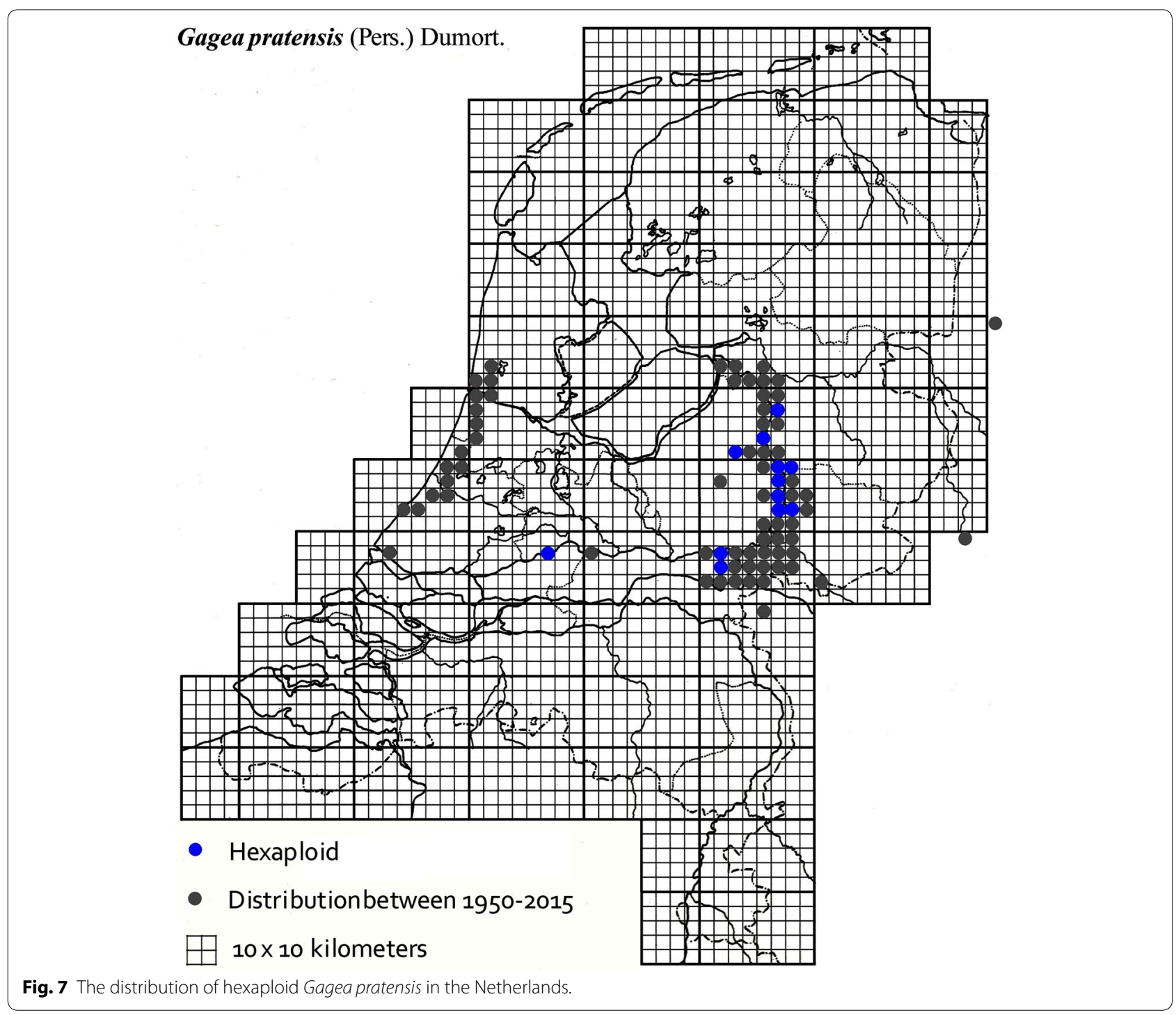

\section{Conclusions}

Five species and different inferred ploidies are recorded for The Netherlands, as summarized in Table 2, some of the latter for the first time. Inferred decaploidy in $G$. pratensis was not demonstrated earlier. G. minima has an inferred diploid size. G. minima was only recently (1994) recognized as a new species for the Netherlands (Diemeer 2005). It is not clear whether it reached Haarlem by itself or was imported with lime trees from abroad. After all, Linnaeus lived there for 3 years only a kilometer away. The nearest known locality is $300 \mathrm{~km}$ away in Germany. G. pratensis is inferred to have four cytotypes: tetraploid, pentaploid, hexaploid and decaploid. Remarkable is the high number, 85 out of 186 accessions, of the pentaploid cytotype. Although it is largely sterile, bulbs seem to be a very effective way for vegetative multiplication, just as found for G. spathacea (Pfeiffer et al. 2012). Gagea lutea is only found in an inferred hexaploid form. The nonaploidy reported for G. spathacea would suggests a low basic genome size. This is corroborated by the fact that G. spathacea belongs to a section different from the others. Flow cytometry could provide the correct identification in most cases. It is a taxonomic and diagnostic tool that is applicable even in the case of dormant bulbs or sterile plants, and therefore has applications for conservation monitoring. Future research of the Dutch gageas could focus on combining chromosome counts and flow cytometry of the same samples, especially in the case of G. villosa. The fertility of the pentaploid G. pratensis 


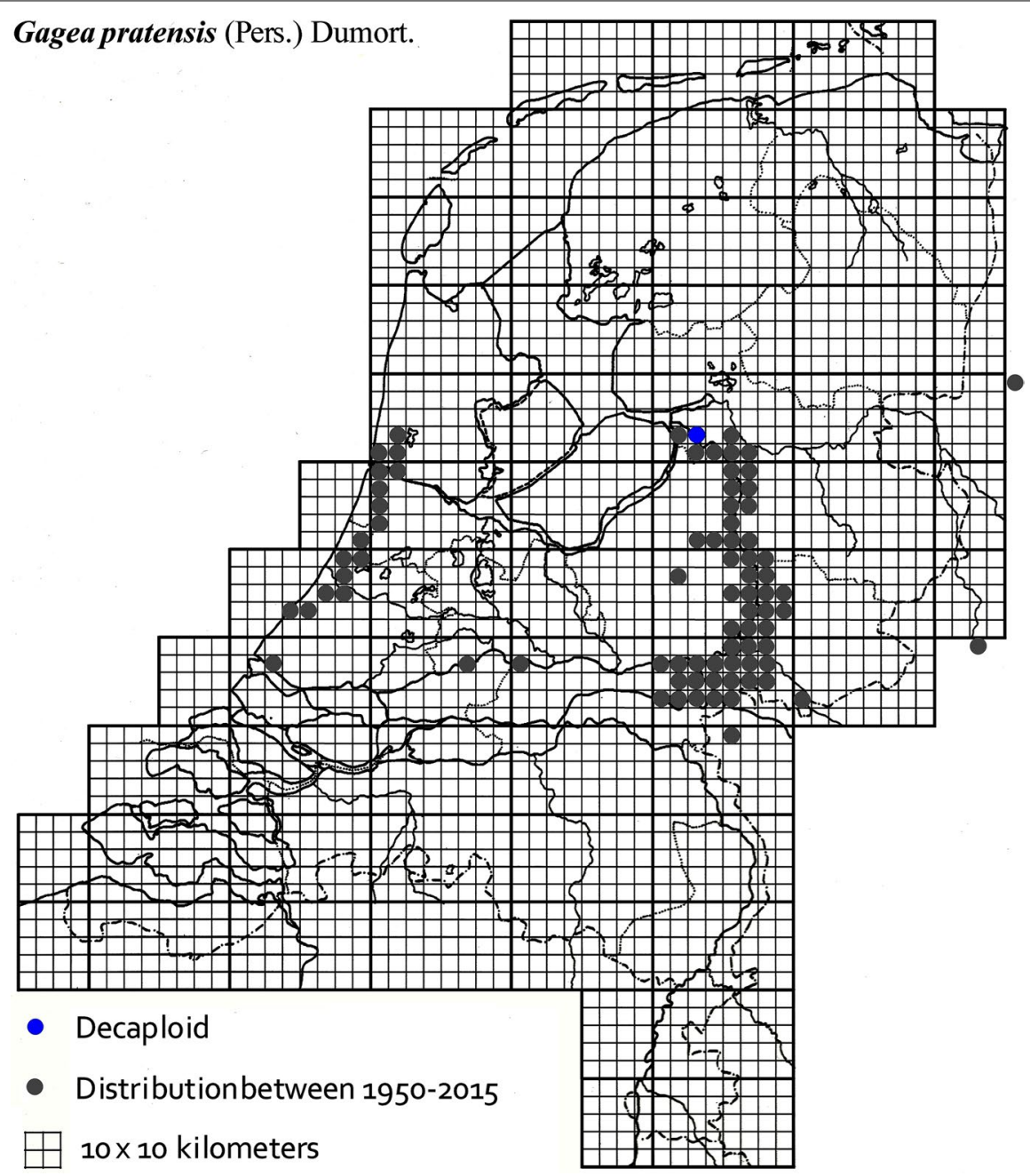

Fig. 8 The distribution of decaploid Gagea pratensis in the Netherlands.

Table 2 Summary of genome sizes in pg (2C), number of accessions and inferred chromosome numbers of species of Dutch and German gageas

\begin{tabular}{|c|c|c|c|c|}
\hline Species & Average $\mathrm{pg} / 2 \mathrm{C}$ & Inferred ploidy & Chromosome number & Number of accessions \\
\hline Gagea minima (L.) Ker Gawl. & 14.9 & Diploid & $2 x=24$ & 2 \\
\hline Gagea villosa (M.Bieb.) Sweet & 17.0 & Diploid & $2 x=24$ & 14 \\
\hline Gagea villosa (M.Bieb.) Sweet & 32.3 & Tetraploid & $4 x=48$ & 6 \\
\hline Gagea lutea (L.) Ker Gawl. & 42.7 & Hexaploid & $6 x=72$ & 22 \\
\hline Gagea lutea var. glauca L.Klein & 42.3 & Hexaploid & $6 x=72$ & 16 \\
\hline Gagea spathacea (Hayne) Salisb. & 46.7 & Nonaploid & $9 x=108$ & 17 \\
\hline Gagea pratensis (Pers.) Dumet. & 32.8 & Tetraploid & $4 x=48$ & 30 \\
\hline Gagea pratensis (Pers.) Dumet. & 39.9 & Pentaploid & $5 x=60$ & 85 \\
\hline Gagea pratensis (Pers.) Dumet. & 45.6 & Hexaploid & $6 x=72$ & 20 \\
\hline Gagea pratensis (Pers.) Dumet. & 75.8 & Decaploid & $10 x=120$ & 2 \\
\hline G. $\times$ pomeranica R.Ruthe & 34.9 & Hexaploid & $5 x=60$ & 11 \\
\hline G. $\times$ megapolitana Henker & 46.8 & Hexaploid & $6 x=72$ & 2 \\
\hline
\end{tabular}

The term 'inferred ploidy' indicates that the ploidy is derived from the genome size and not based on chromosome counts. It is preferred to the proposed term 'DNA ploidy' (Suda et al. 2006) as this seems more ambiguous. Inferred decaploidy is found for the first time in G. pratensis. The hybrid $G$. $\times$ megapolitana, is only collected in Germany so far. The largest genome contains roughly $60 \times 10^{9}$ more base pairs than the smallest. A difference of $1 \mathrm{pg}$ amounts to a difference of nearly $1 \times 10^{9}$ base pairs, so far exceeds a single taxonomic character. 


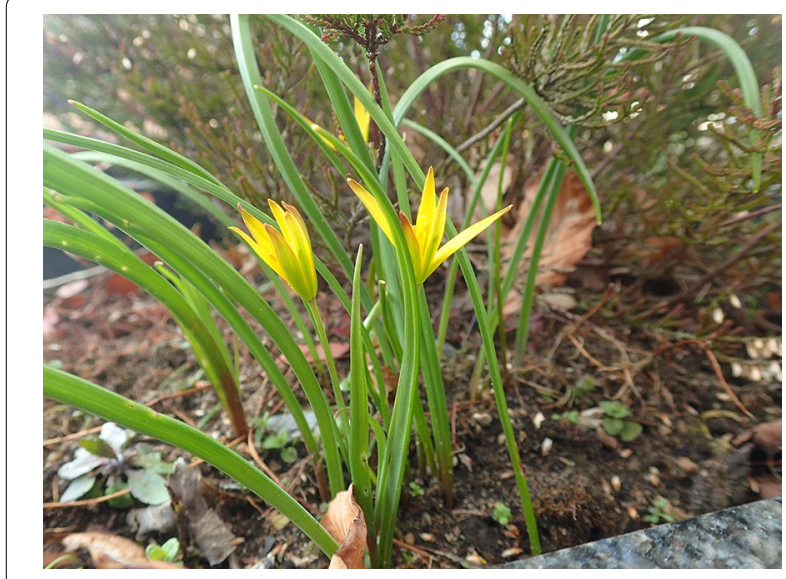

Fig. 9 Gagea pratensis tetraploid April 6, 2015 in a churchyard in Beek.

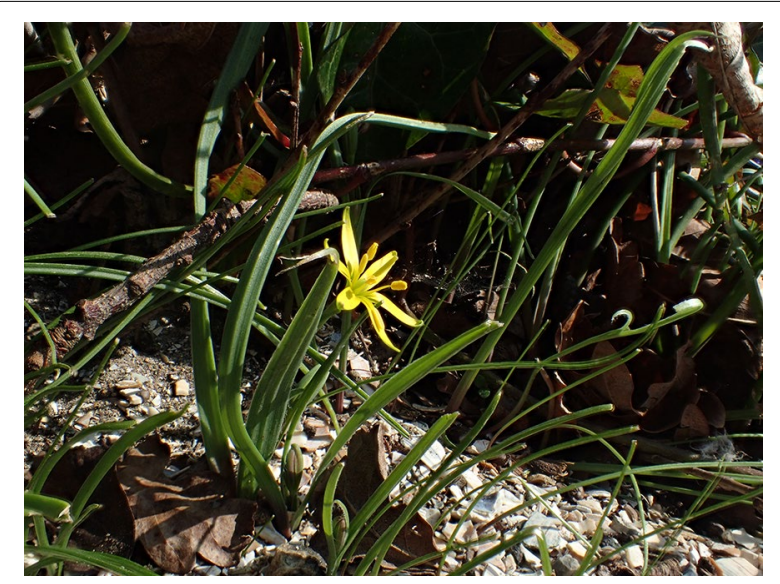

Fig. 10 Gagea pratensis pentaploid (a) March 17, 2015 in a churchyard in Wassenaar.

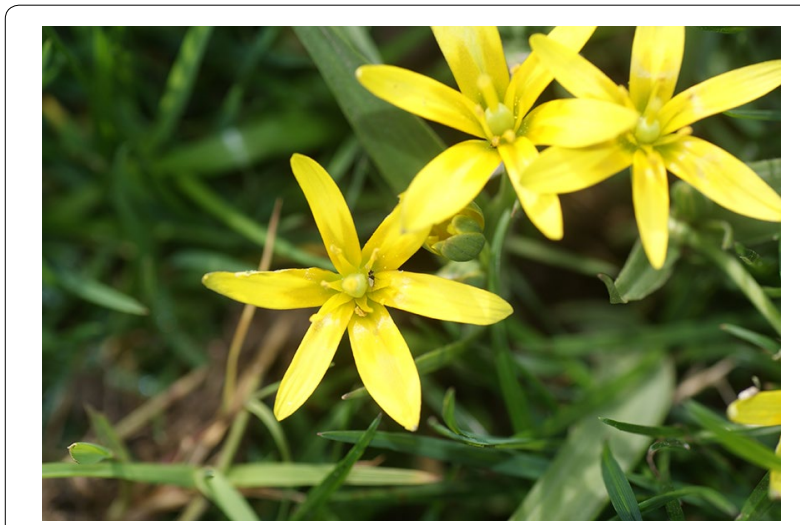

Fig. 11 Gagea pratensis pentaploid (b) April 3, 2011 in Bingerden.

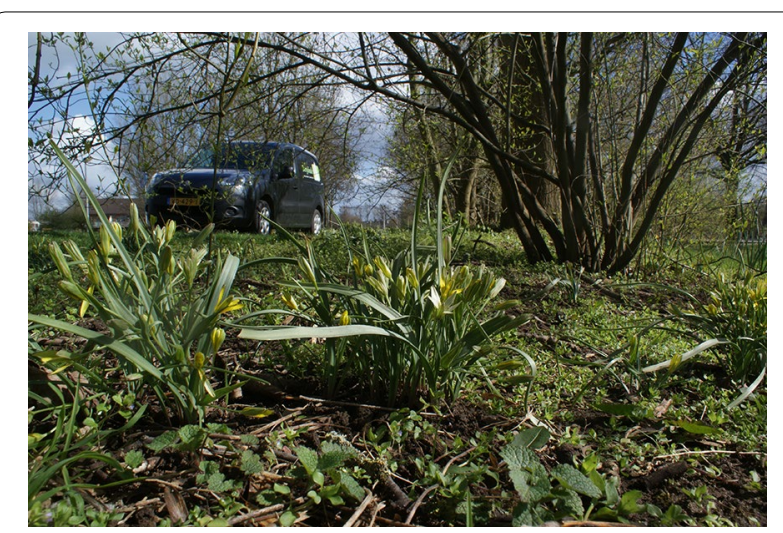

Fig. 12 Gagea pratensis pentaploid (c) March 23, 2014 in a park in Zutphen.

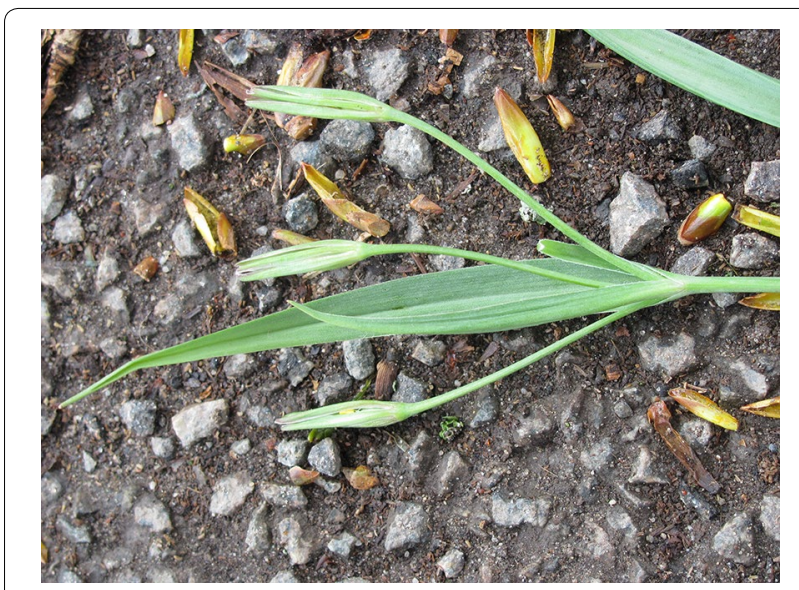

Fig. 13 Gagea pratensis hexaploid April 22, 2011 in a road verge in Brummen.

needs further investigation. Sequencing of the forma glauca of G. lutea could reveal if it is a separate species or not.

\section{Authors' contributions}

BtL and LJB collected the plants, provided morphological and biogeographical data and corrected the manuscript. BZ did the flow cytometry and drafted the manuscript. BtL provided and made all figures. All authors read and approved the final manuscript.

\section{Author details}

${ }^{1}$ NBC Naturalis, Herbarium Section, P.O. Box 9517, 2300 RA Leiden, The Netherlands. ${ }^{2}$ Berglinde BV, Dorpstraat 50, 6909 AL Babberich, The Netherlands.

\section{Acknowledgements}

I like to thank Tanja Pfeiffer for providing most of the German material.

\section{Compliance with ethical guidelines}

\section{Competing interests}

The authors declare that they have no competing interests. 
Received: 21 January 2015 Accepted: 17 July 2015

Published online: 05 August 2015

\section{References}

Diemeer J (2005) Gagea minima (L.) Ker-Gawl Nieuw in Nederland-een erfenis van Linnaeus? Gorteria 31:11-18

Doležel J, Bartos J, Voglmayer H, Greilhuber J (2003) Nuclear DNA content and genome size of trout and human. Cytom Part A 51:127-128

Govaerts R (2006) World checklist of Liliaceae. The Board of Trustees of the Royal Botanic Gardens, Kew. Published on the Internet: http://www.kew. org/wcsp/monocots/

Greilhuber J (1979) Evolutionary changes of DNA and heterochromatin amounts in the Scilla bifolia group (Liliaceae). Plant Syst Evol Suppl 2:263-280

Greilhuber J, Ebert I, Lorenz A, Vyskot B (2000) Origin of facultative heterochromatin in the endosperm of Gagea lutea (Liliaceae). Protoplasma 212:217-226

Greilhuber J (2005) Intraspecific variation in genome size in angiosperms, identifying its existence. Ann Bot 95:91-98

Greilhuber J, Borsch T, Muller K, Worberg A, Porembski S, Barthlott W (2006) Smallest angiosperm genomes found in Lentibulariaceae with chromosomes of bacterial size. Plant Biol 95:255-260

Henker H (2005) Die Goldsterne from Mecklenburg-Vorpommern unter besonderen Berucksichtigung kritischer und neuer Sippen. Botanischer Rundbrief fur Mecklenburg-Vorpommern 39:5-89

Henker H, Kiesewetter H, Raabe U, Ratzel S (2012) Der Markischen Goldstern (Gagea marchica spec nov)-ein neue Sippe aus dem Gagea pomeranica complex. Botanischer Rundbrief fur Mecklenburg-Vorpommern 49:3-12

Leitch IJ, Beaulieu JM, Cheung K, Hanson L, Lysak M, Fay MF (2007) Punctuated genome size evolution in Liliaceae. J Evol Biol 20:2296-2308

Ohri D (1998) Genome size variation and plant systematics. Ann Bot 82(Suppl A):750-812

Pellicer J, Fay MF, Leitch IJ (2010) The largest eukaryotic genome of them all? Bot J Linn Soc 164(1):10. doi:10.1111/j.1095-8339.2010.01072

Peruzzi L (2003) Contribution to the cytotaxonomical knowledge of Gagea Salisb (Liliaceaea) section Foliatae and synthesis of karyological data. Caryologia 56(1):115-128

Peruzzi L (2012) Chromosome diversity and evolution in the genus Gagea (Liliaceae). Bocconea 24:147-158

Peterson A, Levichev IG, Peterson J (2008) Systematics of Gagea and Lloydia(Liliaceae) and infrageneric classification of Gagea based on molecular and morphological data. Mol Phyl Evol 26:446-465

Peterson A, Harpke D, Peruzzi L, Levichev IG, Tison JM, Peterson J (2009) Hybridisation drives speciation in Gagea (Liliaceae). Plant Syst Evol 278:133-148

Pfeiffer T, Klahr A, Heinrich A, Schnittler M (2011) Does sex makes a difference? Genetic diversity and spatial genetic structure in two co-occurring species of Gagea (Liliacae) with contrasting reproductive strategies. Plant Syst Evol 292:189-201

Pfeiffer T, Klahr A, Peterson A, Levichev IG, Schnittler M (2012) No sex at all? Extremely low genetic diversity in Gagea spathacea (Liliaceae) across Europe. Flora Morphol Distrib Funct Ecol Plants 207(5):372-378
Pfeiffer T, Harter DEV, Formella N, Schnittler M (2013) Reproductive isolation vs inbreeding between Gagea lutea(L)Ker Gawl and G. pratensis(Pers) Dumort (Liliaceae) and their putative hybrids in Mecklenburg-Western Pomerania (Germany). Plant Spec Biol 28:193-203

Suda J, Krahulcova A, Travniek P, Krahulec F (2006) Ploidy level versus DNA ploidy level: an appeal for consistent terminology. Taxon 55:447-450

Tiersch TR, Chandler RW, Wachtel SSM, Ellias S (1989) Reference standards for flow cytometry and application in comparative studies of nuclear DNA content. Cytometry 10:706-710

van den Berg LJ, te Linde B (2003) Geelsterren in Gelderland. Afdeling landelijk gebied, Provincie Gelderland, pp 1-28

van Oostrom SJ, Reichgelt TJ (1964) Flora Neerlandica, Deel 1, Liliaceae, Gagea. In: KNBV, van Oostrom SJ et al (eds), 1(6):97-108

van der Meijden R (2005) Heukels'Flora van Nederland. Ed 23 Wolters-Noordhoff, Groningen, Nederland

Vesely P, Bures P, Smarda P, Pavlicek T (2011) Genome size and DNA base composition of geophytes: the mirror of phenology and ecology? Ann Bot 109:65-75

Westergaard M (1936) A cytological study of Gagea spathacea with a note on the chromosome number and embryo-sac formation in Gagea minima. Comp Rend Trav Lab Carlsb Ser Phys 21:437-451

Zarrei M, Wilkin P, Fay MF, Ingrouille MJ, Zarre S, Chase MW (2009) Molecular systematics of Gagea and Lloydia (Liliaceae, Liliales), implications of analysis of nuclear ribosomal and plastid DNA sequences for infrageneric classifications. Ann Bot 104:125-142

Zonneveld BJM (2001) Nuclear DNA contents of all species of Helleborus discriminate between species and sectional divisions. Plant Syst Evol 229:125-130

Zonneveld BJM (2003) The systematic value of nuclear DNA content in Clivia. Herbertia 57:41-47

Zonneveld BJM (2008) The systematic value of nuclear DNA content for all species of Narcissus L. (Amaryllidaceae). Plant Syst Evol 275:109-132

Zonneveld BJM (2009) The systematic value of nuclear genome size for all species of Tulipa L. (Liliaceae). Plant Syst Evol 281:217-245

Zonneveld BJM (2010) New record holders for maximum genome size in eudicots and monocots. J Bot 2010:527357. doi:10.1155/2010/527357

Zonneveld BJM, Van Iren F (2001) Genome size and pollen viability as taxonomic criteria, application to the genus Hosta. Plant Biol 3:176-185

Zonneveld BJM, Duncan GD (2003) Taxonomic implications of genome size and pollen color and vitality for species of Agapanthus L'Héritier (Agapanthaceae). Plant Syst Evol 241:115-123

Zonneveld BJM, Duncan GD (2006) Genome size for the species of Nerine Herb. (Amaryllidaceae) and its evident correlation with growth cycle, leaf width and other morphological characters. Plant Syst Evol 257:251-260

Zonneveld BJM, Grimshaw JM, Davis AP (2003) The systematic value of nuclear DNA content in Galanthus. Plant Syst Evol 241:89-102

Zonneveld BJM, Pollock WI (2012) Sports and hybrids of triploid Hosta'Sum and Substance' reveal chromosome losses and gains in all three apical layers. Plant Syst Evol 298:1037-1043

\section{Submit your manuscript to a SpringerOpen ${ }^{\odot}$ journal and benefit from:}

- Convenient online submission

- Rigorous peer review

- Immediate publication on acceptance

- Open access: articles freely available online

- High visibility within the field

- Retaining the copyright to your article

Submit your next manuscript at $>$ springeropen.com 